\title{
Amiloidose com expressão em cavidade bucal: relato de caso
}

\author{
Amyloidosis with expression in oral cavity: case report
}

\author{
Larissa Araújo Queiroz \\ Thiago Marcelino Sodré** \\ Juliana Andrade Cardoso ${ }^{* * *}$ \\ Lívia Pugliese $e^{* * *}$ \\ Antônio Varela Cancio ${ }^{* * * * *}$ \\ Jener Gonçalves de Farias ${ }^{* * * * *}$
}

\section{Resumo}

Objetivo: o objetivo deste trabalho é relatar um caso raro de amiloidose com expressão em cavidade bucal, discutindo a construção do método diagnóstico do caso com dados da literatura especializada, bem como alertar os profissionais de saúde do seu prognóstico desfavorável e da necessidade de diagnóstico precoce. Relato de caso: paciente do gênero masculino, 87 anos de idade, procurou a clínica-escola de cirurgia do Curso de Odontologia da Universidade Estadual de Feira de Santana (Uefs) no estado da Bahia, referindo como queixa principal um aumento de volume na lateral da língua. No exame físico intrabucal, notaram-se múltiplas lesões nodulares em língua (bilateralmente), moles à palpação, sésseis, de crescimento exofítico, superfície rugosa e coloração esbranquiçada. O diagnóstico histopatológico pela coloração hematoxilina-eosina foi inconclusivo, porém, o patologista bucal observou a presença de depósitos de proteínas. Foi, então, realizado o exame histoquímico vermelho congo, que confirmou a presença de depósitos de material amiloide. Dois meses após o diagnóstico, o paciente foi a óbito, não sendo possível determinar se tratava de uma doença local ou sistêmica. Considerações finais: ressalta-se a importância de o cirurgião-dentista conhecer as manifestações clínicas da amiloidose, assim como participar da equipe multiprofissional para diagnosticar as doenças sistêmicas com envolvimento da cavidade oral.

Palavras-chave: Amiloidose. Vermelho congo. Língua.

\section{Introdução}

Amiloidose é uma designação genérica ${ }^{1}$ para a deposição extracelular anormal de uma substância rica em proteínas (insolúvel e resistente à proteólise) na pele ou em outros órgãos, podendo ocorrer em qualquer órgão do corpo ${ }^{2}$. É uma doença complexa ${ }^{3}$ de manifestação sistêmica ou local ${ }^{4}$. Sua manifestação sistêmica pode ser primária, associada ao mieloma, ou secundária, associada à hemodiálise e à hereditariedade. Quando a manifestação é localizada, apresenta apenas envolvimento cutâneo ${ }^{2}$.

A amiloidose apresenta muitos subtipos, que variam amplamente em suas estratégias terapêuticas $^{5}$, curso clínico e prognóstico, tornando-se imperativo para os clínicos estabelecer um correto diagnóstico precoce antes de traçar a terapêutica ${ }^{6}$. Contudo, a razão para a deposição localizada ainda é desconhecida ${ }^{7}$. Provavelmente inúmeros fatores atuam $^{8}$, mas sabe-se que não há correlação definida com tabagismo, abuso vocal e infecções recorrentes ${ }^{7}$.

Apresenta-se com ampla variedade de sinais e sintomas, dependendo dos órgãos acometidos ${ }^{9}$. Dessa forma, suas complicações podem envolver a disfunção de diversos órgãos, promovendo elevada morbimortalidade ${ }^{5}$.

Dificilmente as lesões da amiloidose ocorrem na região de cabeça e pescoço, sendo ainda mais raras em cavidade oral. Quando presentes nessa área,

Graduada em Odontologia pela Universidade Estadual de Feira de Santana (Uefs), Feira de Santana, Bahia, Brasil.

Bolsista de pós-graduação em Saúde da Família pelo UNA-SUS.

Mestre em Estomatologia Clínica pela Pontifícia Universidade Católica do Rio Grande do Sul (PUCRS), especialista em Estomatologia pela União Metropolitana de Educação e Cultura (Unime), professora do Núcleo de Propedêuticas Clínica e Cirúrgica da (Unime), Lauro de Freitas, Bahia.

Doutora em Imunologia pela Universidade Federal de São Paulo (Unifesp), mestre em Imunologia pela Universidade Federal da Bahia (UFBA), especialista em Patologia Bucal pelo Centro de Pós-Graduação São Leopoldo Mandic, professora do Departamento de Ciências da Vida da Universidade Estadual da Bahia (Uneb), Salvador, Bahia.

***** Mestre em Odontologia pela Universidade Federal da Bahia (UFBA), especialista em Cirurgia e Traumatologia Bucomaxilofacial pela Universidade de Pernambuco (UPE), professor substituto de Cirurgia e Traumatologia Bucomaxilofacial da Universidade Estadual de Feira de Santana (Uefs), Feira de Santana, Bahia.

${ }_{* * * * * *}$ Doutor em Estomatologia pela Universidade Federal da Paraíba (UFPB), mestre em Cirurgia e Traumatologia Bucomaxilofacial pela Universidade de Pernambuco (UPE), professor titular do Curso de Odontologia da Universidade Estadual de Feira de Santana (Uefs), Feira de Santana, Bahia, professor do Núcleo de Propedêuticas Clínica e Cirúrgica da União Metropolitana de Educação e Cultura (Unime), Lauro de Freitas, Bahia. 
afetam mais frequentemente a laringe e a tireoide ${ }^{3}$. $\mathrm{Na}$ boca, a língua é o sítio anatômico mais envolvido, entre $12 \%$ e $20 \%$ dos casos. A cor das membranas mucosas das lesões intraorais pode variar entre amarelo, laranja, vermelho, azul e roxo, e, geralmente, as lesões estão representadas na forma de nódulos, pápulas ou placas ${ }^{3}$, podendo haver ulcerações, fissuras, hemorragias e marcas de dentes lateralmente à língua ${ }^{2}$ O segundo sítio em cavidade oral mais atingido é o lábio².

Portanto, devido à diversidade de sintomas, basear o diagnóstico exclusivamente nas manifestações clínicas é imprudente ${ }^{6}$, e a biópsia tecidual deve ser realizada para o diagnóstico definitivo ${ }^{3}$. Entretanto, existem colorações mais específicas que a coloração de hematoxilina-eosina (HE), que facilitam a observação, como o vermelho congo, o vermelho sirius e a violeta de genciana ${ }^{8}$.

A avaliação sistêmica desses pacientes deve sempre ser realizada para descartar o envolvimento de outros órgãos pela amiloidose ${ }^{10}$. Preferencialmente, a biópsia deve ser direcionada para o órgão mais envolvido clinicamente ${ }^{11}$, e algumas técnicas de imagem, como a tomografia computadorizada, a ressonância magnética nuclear e a cintilografia, podem ser de grande utilidade ${ }^{12}$.

A escolha do tratamento depende do tipo de amiloidose. Todo planejamento deverá encaminhar-se para dois objetivos: diminuição da proteína precursora do tipo amiloide em questão e terapia de suporte para o órgão acometido ${ }^{3}$.

O objetivo deste trabalho é relatar um caso raro de amiloidose com expressão em cavidade bucal, discutindo a construção do método diagnóstico do caso com dados da literatura especializada, bem como alertar a comunidade da necessidade do diagnóstico precoce devido ao prognóstico que a enfermidade apresenta.

\section{Relato de caso}

Paciente masculino, 87 anos, procurou a clínica-escola de cirurgia do Curso de Odontologia da Universidade Estadual de Feira de Santana (Uefs) no estado da Bahia, em outubro de 2011, referindo como queixa principal o aumento de volume na lateral da língua. $\mathrm{Na}$ história médica, relatou ter hipertensão e arritmia cardíaca e ter tido câncer no duodeno, informações que foram verificadas por meio do relatório médico apresentado pelo paciente.

No exame físico intrabucal, foram observadas múltiplas lesões nodulares em língua (bilateralmente), moles à palpação, sésseis, de crescimento exofítico, superfície rugosa e coloração esbranquiçada (Figuras 1 e 2).

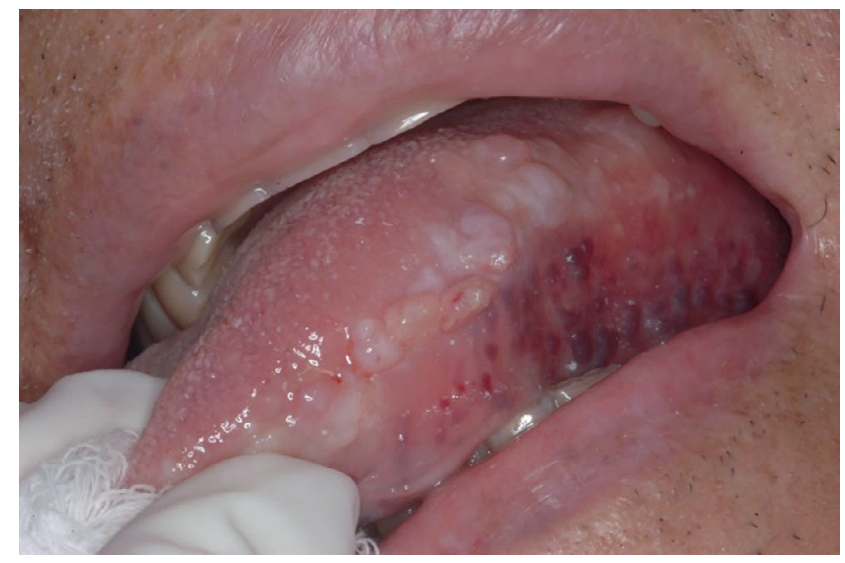

Figura 1 - Aspecto clínico bordo lateral esquerdo de língua, evidenciando múltiplas lesões nodulares de coloração esbranquiçada, base séssil, superfície rugosa e consistência compressivel

Fonte: dos autores.

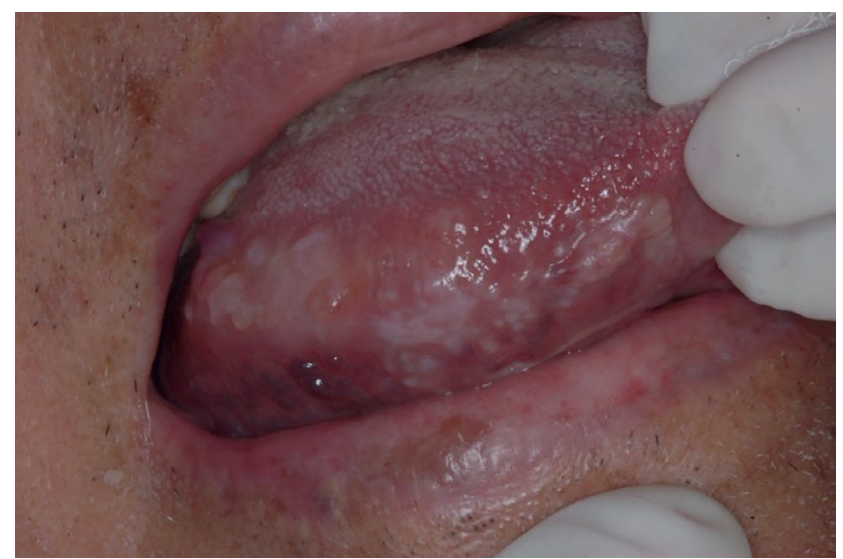

Figura 2 - Aspecto clínico bordo lateral direito de língua, evidenciando múltiplas lesões nodulares de coloração esbranquiçada, base séssil, superfície rugosa e consistência compressível

Fonte: dos autores.

De acordo com as características clínicas apresentadas, as hipóteses diagnósticas foram linfangioma ou amiloidose. Optou-se pela realização de biópsia incisional (Figura 3).

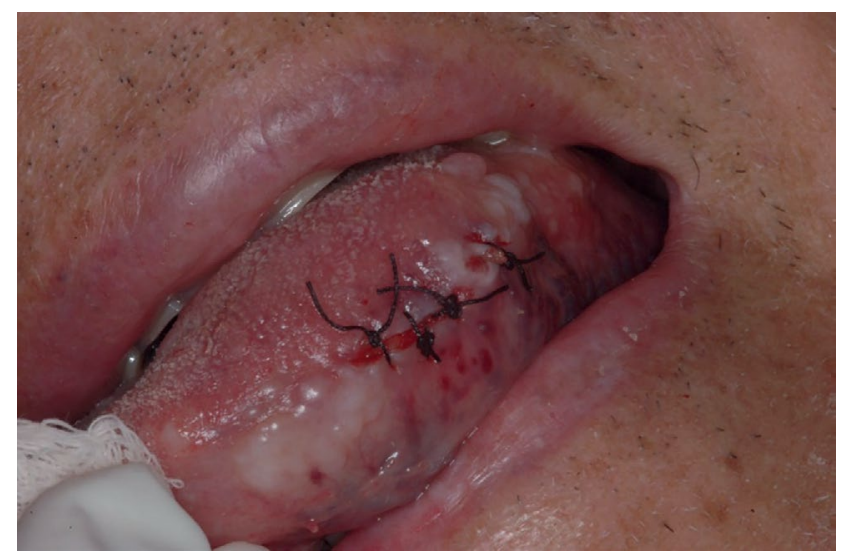

Figura 3 - Aspecto da sutura imediatamente após realização de biópsia incisional

Fonte: dos autores. 
As peças foram encaminhadas para estudo anatomopatológico (Figura 4).

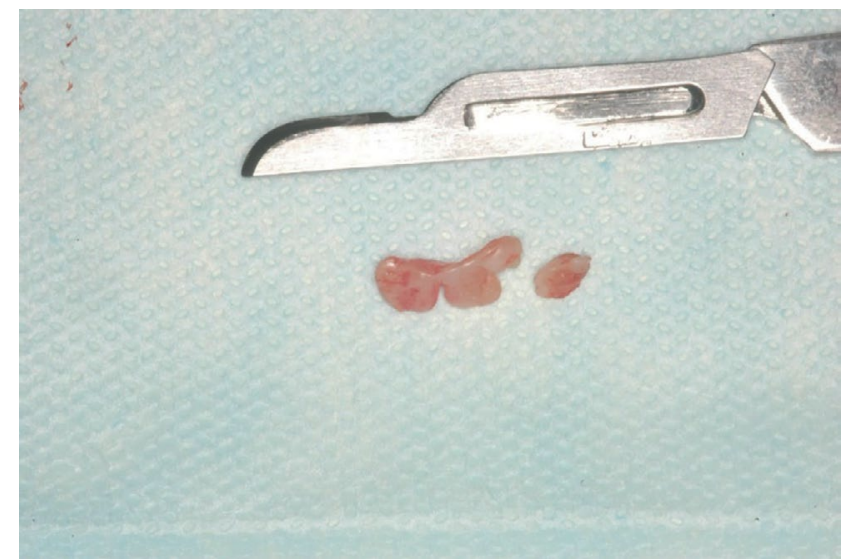

Figura 4 - Peças cirúrgicas removidas por meio de biópsia incisional, encaminhadas para exame anatomopatológico

Fonte: dos autores.

O exame macroscópico mostrou fragmentos de tecido mole, de consistência fibroelástica, formato irregular e superfície multinodular, medindo, o maior, $1,1 \times 0,5 \times 0,4 \mathrm{~cm} \mathrm{e,} \mathrm{o} \mathrm{menor,} 0,3 \times 0,3 \times 0,3 \mathrm{~cm}$.

$\mathrm{O}$ diagnóstico histopatológico inicial com a coloração HE revelou uma mucosa escamosa com acantose e áreas de hiperqueratose, apresentando ulceração e tecido conjuntivo subjacente com intenso infiltrado inflamatório misto e deposição de material eosinofílico (Figuras 5 e 6).

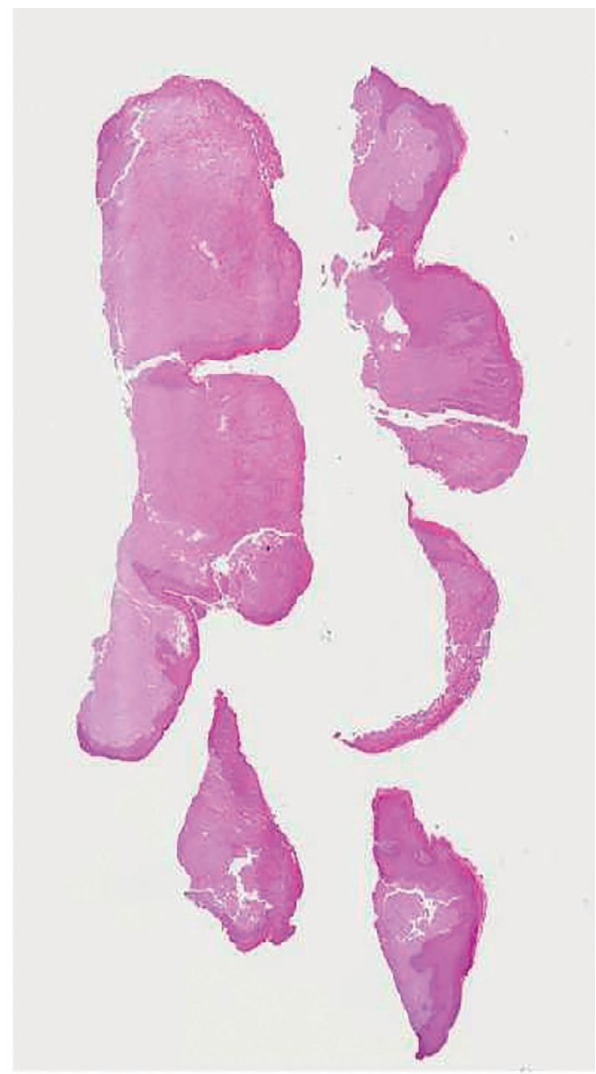

Figura 5 - Aspecto histopatológico

Fonte: dos autores.

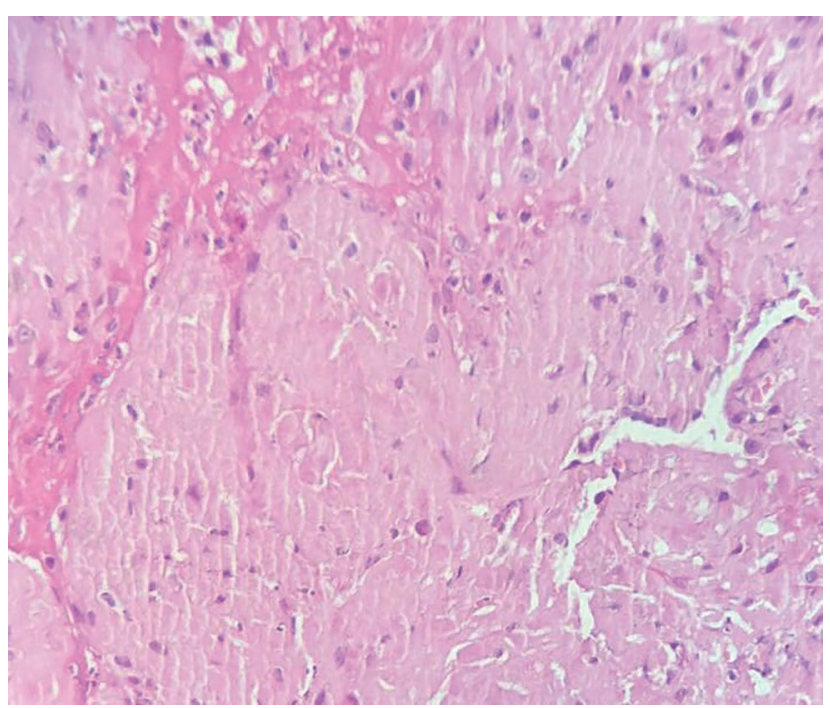

Figura 6 - Aspecto histológico revelando tecido conjuntivo com intenso infiltrado inflamatório misto e deposição de material eosinofílico com coloração positiva para vermelho congo

Fonte: dos autores.

O diagnóstico histopatológico pela coloração HE foi inconclusivo, porém, o patologista bucal observou a presença de depósitos de proteínas. Foi, então, realizado o exame histoquímico vermelho congo, que é específico para definição de amiloidose, o que confirmou a presença de depósitos de amiloide (Figuras 7 e 8).

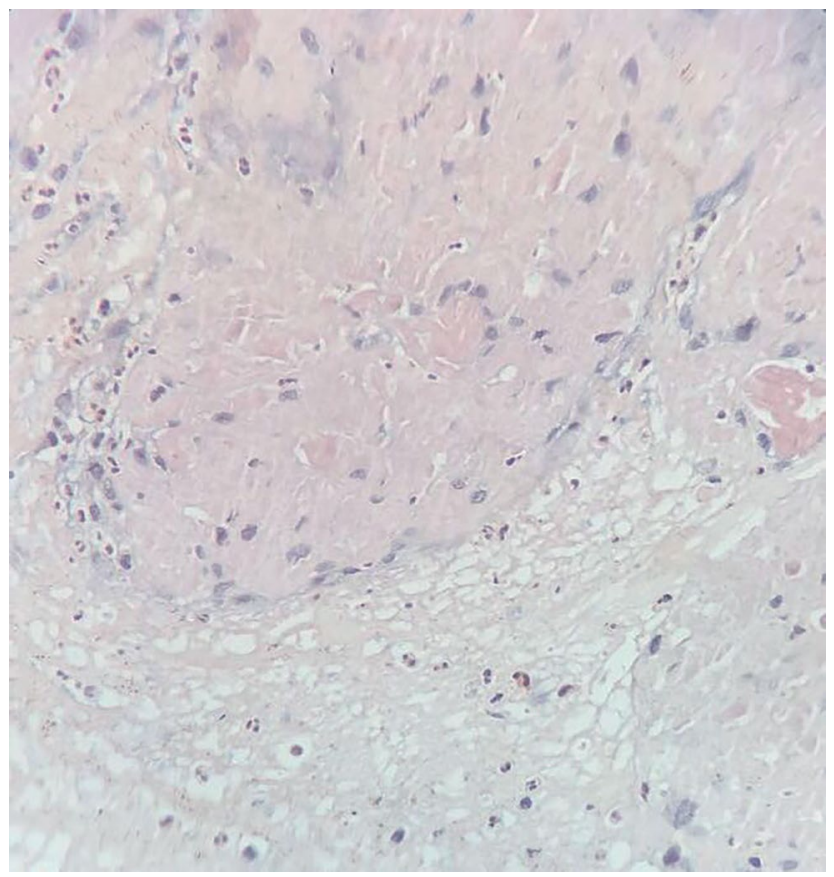

Figura 7 - Imagem da lâmina com coloração vermelho congo observada em microscopia de luz convencional, evidenciando deposição de material eosinofílico (Objetiva 10 X).

Fonte: dos autores. 


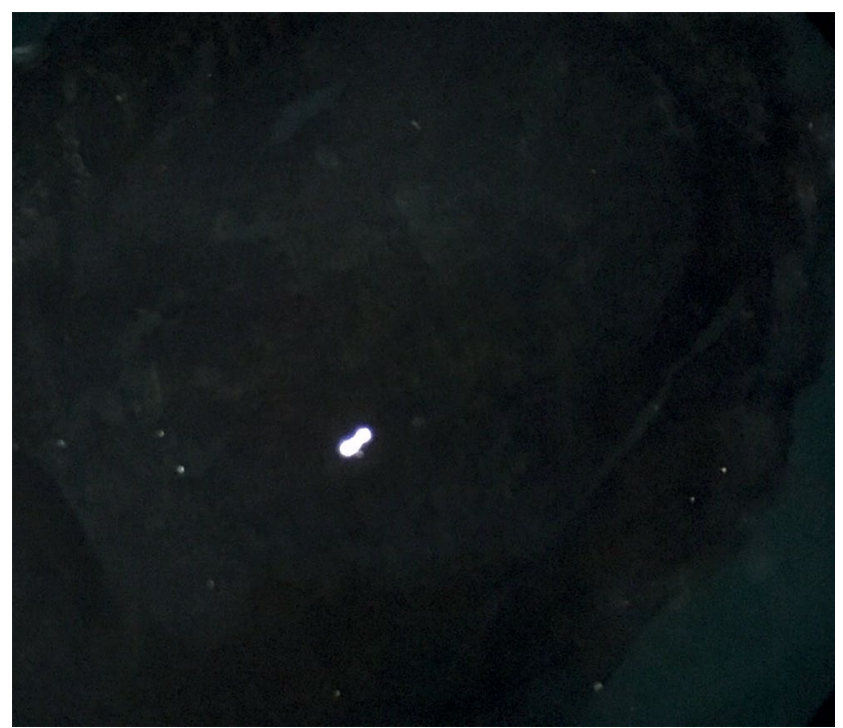

Figura 8 - Imagem da lâmina com coloração vermelho congo observada na luz polarizada, evidenciando coloração esverdeada no depósito eosinofílico (Objetiva 10 X).

Fonte: dos autores.

Dessa forma, na tentativa de avaliar envolvimento sistêmico, foram solicitados exames complementares básicos (hemograma e sumário de urina), em que o paciente apresentou proteinúria de quatro cruzes, por isso foi encaminhado para atendimento médico. No entanto, dois meses após o diagnóstico, o paciente foi a óbito, não sendo possível determinar se se tratava de uma doença local ou sistêmica.

\section{Discussão}

A prevalência da amiloidose aumenta com a idade: $1,7 \%$ nos pacientes com idade entre 50 a 59 anos e mais de $5 \%$ naqueles com mais de 70 anos, sendo as taxas de prevalência maior em homens do que em mulheres ${ }^{13}$. Esse dado está de acordo com $o$ relato apresentado, pois se trata de um homem com mais de 80 anos de idade.

No exame físico intrabucal, observaram-se múltiplas lesões nodulares em língua (bilateralmente), moles à palpação, sésseis, de crescimento exofítico, superfície rugosa e coloração esbranquiçada. Essa característica corrobora a literatura, que evidencia que a amiloidose, quando se expressa em cavidade bucal, geralmente acomete a língua e apresenta-se como nódulos sésseis de coloração esbranquiçada ${ }^{3}$.

$\mathrm{O}$ paciente relatou, como queixa principal, o aumento de volume na borda lateral da língua. Essa sintomatologia está de acordo com a literatura, que prevê que a deposição de material amiloide na língua ocorre com frequência e pode resultar em macroglossia (aumento do volume da língua), achado oral mais frequente nesses casos ${ }^{14}$. O primeiro sinal de amiloidose primária pode ser o aumento de volume da língua ${ }^{15}$.

Geralmente, a amiloidose se comporta de uma maneira inofensiva, quando é localizada na região de cabeça e pescoço ${ }^{13}$, contudo, em todas as formas de amiloidose sistêmica, a evolução do paciente é geralmente contínua, grave e, finalmente, fatal. A resposta do organismo aos tratamentos dos quadros de amiloidose é difícil, e a sobrevida é bastante reduzida em pacientes portadores de amiloidose sistêmica ${ }^{3}$.

A maioria dos pacientes morre de falência cardíaca, arritmia, ou de doença renal dentro de alguns meses ou poucos anos após o diagnóstico ${ }^{14}$. Essa constatação pode ser associada ao caso, visto que o paciente era hipertenso e possuía arritmia cardíaca, quadros possíveis da causa da morte.

O diagnóstico histopatológico inicial com a coloração $\mathrm{HE}$ revelou uma mucosa escamosa com acantose e áreas de hiperqueratose, apresentando ulceração e tecido conjuntivo subjacente com intenso infiltrado inflamatório misto e deposição de material eosinofílico. O diagnóstico pela coloração HE foi inconclusivo. Porém, após observar presença de depósitos de proteínas, foi realizado o exame histoquímico vermelho congo, que é específico para definição de amiloidose. Esta coloração confirmou a presença de depósitos de amiloide. Esses aspectos estão de acordo com a descrição da literatura, que recomenda como método padrão para identificação da amiloidose o exame histoquímico com vermelho congo ${ }^{3}$.

O exame histopatológico tem por objetivo determinar o tipo de amiloidose que o paciente é portador. Frequentemente envolve uma avaliação pela eletroforese sérica para verificar se existe uma gamopatia monoclonal, a fim de confirmar a existência do mieloma múltiplo ${ }^{14}$. No caso relatado, só houve a confirmação após o exame histopatológico com vermelho congo, quando se confirmou o diagnóstico de amiloidose; entretanto, não houve conclusões quanto ao tipo de doença, se local ou sistêmica, por conta do óbito do paciente. Esse dado enfatiza a necessidade do diagnóstico precoce.

De acordo com Xavier et al. $(2004)^{12}$, a biópsia de língua leva ao diagnóstico de amiloidose, porém não define se seu subtipo é a forma sistêmica ou localizada, o que é importante devido à diferença de sobrevida entre uma forma e outra. A forma localizada, extremamente rara, tem prognóstico melhor do que a forma sistêmica. O diagnóstico entre forma sistêmica e localizada de amiloidose pode ser obtido por meio de aspirado de gordura abdominal ${ }^{1}$.

Na maioria dos casos, não há uma terapia efetiva disponível para a amiloidose ${ }^{13}$. $\mathrm{O}$ debridamento cirúrgico dos depósitos de amiloide na língua tem sucesso limitado. Mas, pode-se inferir que o diagnóstico tardio influenciou negativamente o prognóstico do caso, pois o estado de saúde do paciente já estava comprometido. O paciente morava na cidade de Andaraí e só obteve o diagnóstico quando foi atendido na clínica da Uefs, indo a óbito dois meses depois; diante disso, consideramos que: quanto 
mais tarde for obtido o diagnóstico, mais sombrio será o prognóstico.

Este estudo relatou um caso diagnosticado de amiloidose na cavidade bucal, evidenciando como o material proteico é facilmente identificado por meio do exame histoquímico vermelho congo, específico para definição de amiloidose. É importante dizer que o diagnóstico precoce das lesões da cavidade bucal e o acompanhamento são muito importantes para a sobrevida do paciente.

Neste caso, apesar de os exames complementares básicos terem sido solicitados, não foi possível confirmar se a doença era local ou sistêmica, relacionada à insuficiência renal, em virtude do falecimento do paciente após dois meses do diagnóstico, o que determina o estado avançado da doença.

\section{Considerações finais}

Ressalta-se a importância de o cirurgião-dentista conhecer as manifestações clínicas da amiloidose, assim como participar da equipe multiprofissional, para diagnosticar as doenças sistêmicas com envolvimento da cavidade oral.

\section{Abstract}

Purpose: the aim of this study is to report on a rare case of Amyloidosis in the oral cavity and to investigate diagnostic methods through the specialist literature and to alert about the necessity of an early diagnosis. Case report: an 87 years-old man came to the Dentistry Surgery Clinic in the State University of Feira de Santana (Uefs) in the state of Bahia, complaining about volume growth on the side of the tongue. In the intraoral examination was observed multiple nodular lesions in the tongue (bilaterally), with soft consistence, sessile, exophytic growth, rough surface and whitish. The histopathological diagnosis by HE staining was inconclusive, however, the oral pathologist observed the presence of protein deposits. Afterward, it was performed the congo red histochemical examination, which was confirmed the presence of amyloid deposits. However, two months after the diagnosis, the patient died, so it was not possible to determine if it was a local or systemic disease. Final considerations: to emphasize the importance of dentists to know the clinical manifestations of amyloidosis, as well as the participation of the professional dentist in the multidisciplinary team to diagnose systemic diseases, which affect the oral cavity.

Keywords: Amyloidosis. Congo red. Tongue.

\section{Referências}

1. Santos MSF, Soares B, Mendes O, Carvalho CM, Casimiro RF. Amiloidose-mieloma múltiplo apresentando-se como pseudomiopatia. Rev Bras Reumatol 2011; 51(6):648-54.

2. Oliveira EVL, Pozetti ACG, Pozetti EMO, Antonio JR, Michalany NS. Amiloidose sistêmica primária associada ao mieloma múltiplo. An Bras Dermatol 2012; 87(1):119-22.

3. Ribeiro ILA, Junior VLB, Pereira OHG, Rosa MRD, Lira HJM. Amiloidosis bucal oral amyloidosis. Rev Cubana Estomatol 2012; 49(1):63-70.

4. João A, Serrano P, Bártolo E, Neves A, Brandão FM. Amiloidose sistémica associada a mieloma múltiplo. Med Cutan Iber Lat Am 2010; 38(4):157-60.

5. Silva D, Sargento L, Varela MG, Brito D, Lopes MG. Por detrás da síndrome da insuficiência cardíaca: o diagnóstico de amiloidose AL. A propósito de dois casos clínicos. Serviço de Cardiologia I, Hospital de Santa Maria, Centro Hospitalar Lisboa Norte, EPE, Lisboa, Portugal. Rev Port Cardiol 2010; 29(11):1751-60

6. Moura LBM. Implicações ecocardiográficas na amiloidose cardíaca experts in ultrasound: reviews and perspectives. EURP 2011; 3(4):105-9.

7. Maahs GS, Steffen N, Schwartsmann CC, Trindade VD, Martha VF. Amiloidose laríngea. Revista da AMRIGS 2011; 55(2):173-5

8. Adames GT, Rocha C, Filho SSC, Moreira JR. Líquen amiloidótico: uma manifestação de amiloidose cutânea primária localizada. Rev Med Saude Brasilia 2013; 2(3):156-63.

9. Nobre CA, Callado MRM, Rodrigues CEM, Menezes DB, Vieira WP. Terapia com anti-TNF na amiloidose renal em artrite reumatoide refratária: uma nova perspectiva terapêutica. Rev Bras Reumatol 2010; 50(2):205-10

10. Gonçalves SVCB, Valente NYS, Passaro EMC, Paiva DLM, Dantas FLT, Kakizaki P. Amiloidose nodular com lesão corimbiforme. An Bras Dermatol 2012; 87(5):757-60.

11. Gomes MM. Amiloidose familiar por transtirretina TTR Val30Met e os primórdios do Centro de Estudos de Paramiloidose. Antonio Rodrigues de Mello. Rev Bras Neurol 2011; 47(2):7-21.

12. Xavier SD, Bussoloti Filho I, Muller H. Macroglossia decorrente de amiloidose sistêmica: relato de caso e revisão de literatura. Rev Bras Otorrinolaringol 2004; 70(5):715-9.

13. Babburia S, Ramya B, Subramanyam RV, Aparna V, Gautam Srivastava D. A amiloidose de um caso raro em língua. J Clin Diagn Res 2013; 7(12):3094-5.

14. Bucci T, Bucci E, Rullan AMP, Bucci P, Nuzzolo P. Amiloidose localizada da gengiva superior: relato de caso. J Med Case Rep 2014; 8:198.

15. Kumar V, Abba AK, Jon C. Robbins \& Cotran patologia: bases patológicas das doenças. In: Kumar V, Abba AK, Jon C. Doenças do Sistema Imunológico. 8. ed. Rio de Janeiro, RJ: Elsevier, 2010.
Larissa Araújo Queiroz

Rua Ribeira do Pombal, 80, Núcleo

48770-000, Teofilândia, BA, Brasil

Telefone: (71) 9177-0819

E-mails: larissa07odontonto@gmail.com juliandradec@gmail.com 